\title{
Effects of the Resistivity and Crystal Orientation of the Silicon PIN Detector on the Dark Current and Radiation Response Characteristics
}

\author{
Kun-Sik Park, Jong-Moon Park, Yong-Sun Yoon, Jin-Gun Koo, Bo-Woo Kim, Chang-Joo Yoon, and \\ Kwang-Soo No
}

\begin{abstract}
The effects of the resistivity and crystal orientation on the leakage current and radiation response characteristics have been studied. The detector with (111) oriented substrate shows higher leakage current than (100) orientation due to the higher interface trap density at the $\mathrm{Si} / \mathrm{SiO}_{2}$ interface. And high resistive substrate shows larger leakage current than low resistive one because of its wider depletion width at the same bias voltage. However, in case of (100) oriented substrate, the leakage current of low resistive substrate is larger than high resistive substrate at high reverse bias. It seems that thermionic field emission (TFE) current for low resistive substrate increased at high reverse bias. To compare the charge generation and collection for the radiation, we irradiated an $\mathrm{X}$-ray beam to each detector and read the output current. The detector with (111) oriented substrate shows $20 \%$ higher output current than (100) orientation and it is independent on the resistivity of the substrates. The most influential factor on the output current is the thickness of the wafer. From the results we can suggest a high resistive, (100) oriented and thick wafer for direct type radiation detector, and a low resistive and thin wafer for in-direct type detector. Finally, we assembled our detector with read-out integrated circuit for the application of $\gamma$ ray dosimeter and our detector is very sensitive to $\mathrm{Cs}_{137}$ natural $\gamma$ ray.
\end{abstract}

\section{INTRODUCTION}

SILICON PIN diode is widely used as a radiation detector $S_{\text {because of its low cost, compatibility with the matured }}$ silicon device technology. It is applicable to the multidetectors and large area detectors. And it has great potential to combine with CMOS circuits monolithically and to fabricate the various types of detectors with different wafers in same technology [1].

There are various types of silicon radiation detectors such as discrete, one or two dimensional detector (dosimeter, particle counter, imager, etc.), linear or cylindrical silicon drift detectors (SSDs) for position sensing and X-ray or optical photon detectors and silicon photo-multiplier (SiPM), etc.

Manuscript received November 17, 2006

Kun-Sik Park, Jong-Moon Park, Yong-Sun Yoon, Jin-Gun Koo, and BoWoo Kim are with the Semiconductor Process Team, Electronics and Telecommunications Research Institute, Daejeon, 305-350 Korea (telephone: 82-42-860-6371, e-mail: kunsik@etri.re.kr). Kun-Sik Park is also with the Department of Materials Science and Engineering, Korea Advanced Institute of Science and Technology, Daejeon, 305-701 Korea.

Chang-Joo Yoon is with the Department of Semiconductor Science and Technology, Chonbuk National University, Jeonjoo, 561-756 Korea (telephone: 82-63-270-3651, e-mail: cjyoun@moak.chonbuk.ac.kr).

Kwang-Soo No is with the Department of Materials Science and Engineering, Korea Advanced Institute of Science and Technology, Daejeon, 305-701 Korea (telephone: 82-42-869-4156, e-mail: ksno@kaist.ac.kr).
These detectors are mostly based on the PN or PIN junction diode and thus device structures are simple. However, the specification of the starting wafer (such as resistivity, crystal orientation and wafer thickness, etc.) should be carefully considered depending on their purposes.

Radiation detection is realized by two different methods, i.e. direct type without using scintillator and in-direct type using scintillator on the detector surface. Schematic cross-sectional views of these are described in Fig. 1.

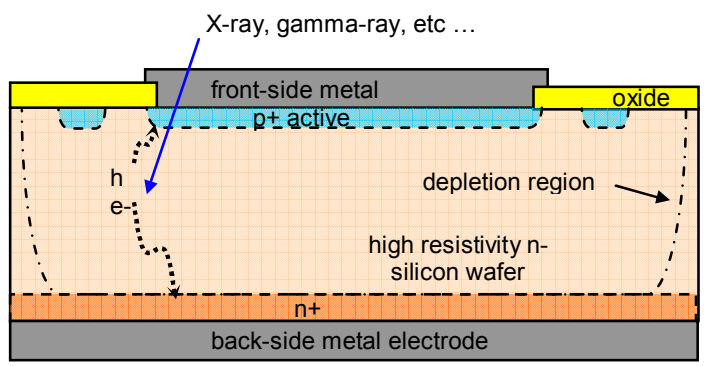

(a)

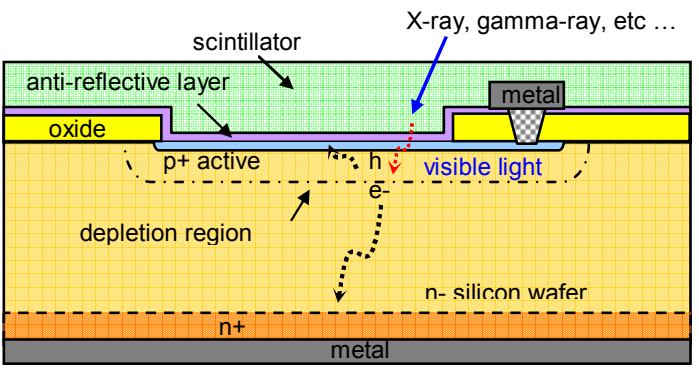

(b)

Fig. 1. Schematic cross-sectional views of (a) direct type silicon radiation detector and (b) in-direct type silicon radiation detector.

Direct type silicon radiation detector is operated at full depletion mode with high operation voltage and several folds of guard-rings are required to improve the breakdown characteristics. On the other hand, in-direct type detector is operated at low reverse bias voltage or $0 \mathrm{~V}$ and the junction depth of the $\mathrm{p}^{+}$active should be shallow to absorb larger quantity of the visible light. Wavelengths of the visible light from scintillator are generally 500 to $600 \mathrm{~nm}$ and a few micron of depletion width is sufficient to absorb this visible light. Then the specification of the starting wafer for these two types of detectors must be different. And different wafers should be 
selected for the different purposes even if the detector type is the same.

In this work we have fabricated radiation detectors with high resistive $n$-type silicon wafers having different resistivity and crystal orientation, and studied on the leakage current, its mechanisms and radiation response characteristics. Then we suggest adequate specification of the wafers for various types of silicon radiation detectors.

\section{FABRICATION OF THE SILICON PIN DETECTORS}

We have fabricated both direct type and in-direct type silicon PIN detectors having $\mathrm{p}+$ active area of $1 \mathrm{~cm} \times 1 \mathrm{~cm}$ with several different n-type FZ (float zone) high resistive silicon wafers. In our experiments, guard-rings are not introduced for both detectors. The specifications of used wafers are summarized in Table I.

TABLE I

SPECIFICATIONS OF STARTING WAFERS. ALL WAFERS ARE PHOSPHOROUS DOPED N-TYPE FLOAT ZONE SILICON WAFERS

\begin{tabular}{c|c|c|c} 
Sample No. & Orientation & Resistivity & Thickness \\
\hline S1 & $(100)$ & $1 \sim 2 \mathrm{k} \Omega . \mathrm{cm}$ & $380 \mu \mathrm{m}$ \\
S2 & $(111)$ & $1 \sim 5 \mathrm{k} \Omega . \mathrm{cm}$ & $525 \mu \mathrm{m}$ \\
S3 & $(111)$ & $>5 \mathrm{k} \Omega . \mathrm{cm}$ & $380 \mu \mathrm{m}$ \\
S4 & $(100)$ & $>5 \mathrm{k} \Omega . \mathrm{cm}$ & $380 \mu \mathrm{m}$
\end{tabular}

The process sequences to fabricate the detectors are as follows. An initial oxide of $500 \mathrm{~nm}$ was grown at the temperature of $900{ }^{\circ} \mathrm{C}$. After removal of initial oxide on the backside of the wafer, $\mathrm{POCl}_{3}$ doping process was carried out to form an $\mathrm{n}+$ layer. In order to form a $\mathrm{p}+$ active region, B11 or $\mathrm{BF}_{2}$ was implanted after removal of initial oxide on the $\mathrm{p}+$ active region. In case of direct type detector, B11 was implanted with $40 \mathrm{keV}, 3 \times 10^{15} \mathrm{~cm}^{-2}$ and driven-in at $900{ }^{\circ} \mathrm{C}$ for $200 \mathrm{~min}$ in nitrogen ambient to deepen and broaden the doping profile of the $\mathrm{p}+$ active region, which can improve the breakdown characteristics of the detectors. On the other hand, the implantation of $\mathrm{BF}_{2}, 40 \mathrm{keV}, 1 \times 10^{15} \mathrm{~cm}^{-2}$ and thermal activation of $900{ }^{\circ} \mathrm{C}, 30 \mathrm{~min}$ were carried out for in-direct type detectors in order to form a shallow junction. In-direct type detectors need some additional processes such as formation of anti-reflective layer and contact hole. A $70 \mathrm{~nm}$ silicon nitride film, which shows the best anti-reflective characteristics for the wavelength of $550 \mathrm{~nm}$, was deposited. Al/TiW double layer metals were deposited to form a front-side metal for anode and a backside metal for cathode. Finally, hydrogen alloy was carried out to make the ohmic contact between metal and silicon and to improve the $\mathrm{Si}-\mathrm{SiO}_{2}$ interface.

We measured several characteristics of the detectors such as junction capacitance, leakage current at various temperature and X-ray response characteristics. To investigate the effects of radiation, we irradiated the proton with energy of $45 \mathrm{MeV}$ and dose of $1 \times 10^{12} \mathrm{~cm}^{-2}$ and compared with non-irradiated samples. Junction capacitance was measured by HP4194A, impedance gain phase analyzer and leakage current was measured by HP4156A, parametric analyzer. X-ray response characteristics was measured by HP4156A with X-ray source having $60 \mathrm{kV}$ of tube voltage, $5 \mathrm{~mA}$ of anode current and filtration of $2 \mathrm{~mm} \mathrm{Al}$.

\section{RESULTS AND ANALYSIS}

\section{A. Junction Capacitance}

We can estimate the resistivity of the wafer and full depletion voltage from the measurement of junction capacitance, $C_{j}$. The doping concentration, $N_{D}$, of the wafers can be calculated from the following two equations

$$
\begin{aligned}
& C_{j}=\left[\frac{q \varepsilon_{s}}{2\left(1 / N_{A}+1 / N_{D}\right)\left(V_{b i}-V_{R}\right)}\right]^{1 / 2} \\
& \text { Slope of } \frac{1}{C_{j}{ }^{2}}-V_{R} \text { Curve }=\frac{2}{q \varepsilon_{s} N_{D}}
\end{aligned}
$$

where $q$ is electronic charge, $\varepsilon_{S}$ is permittivity of silicon, $N_{A}$ is the doping concentration of $\mathrm{p}+$ active $\left(\sim 1 \times 10^{20} \mathrm{~cm}^{-3}\right), V_{b i}$ is built-in potential of the junction and $V_{R}$ is the reverse bias.

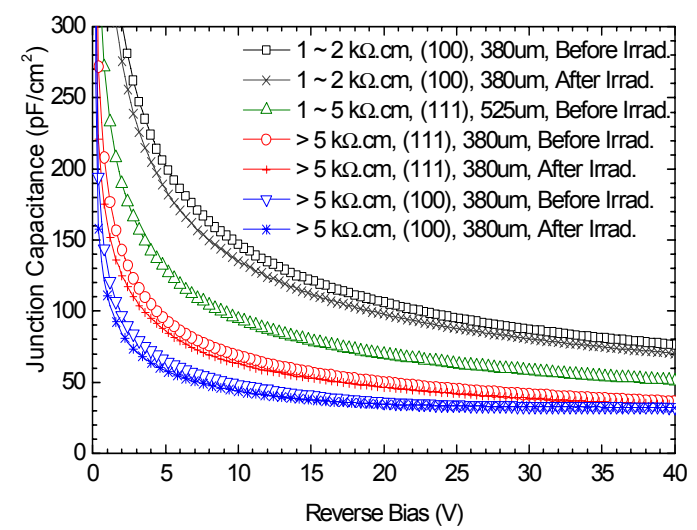

(a)

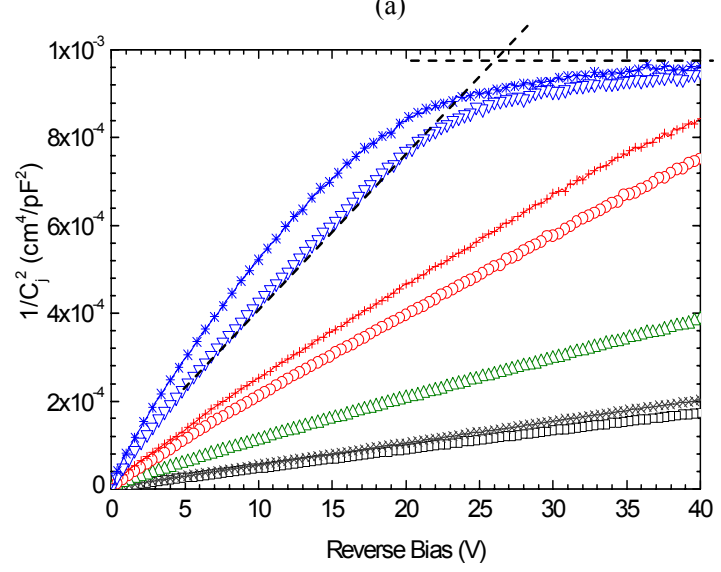

(b)

Fig. 2. Junction capacitance versus reverse bias curves: (a) $C_{j}$ vs. $V_{R}$ and (b) $1 / C_{j}^{2}$ vs. $V_{R}$. The cross point of two dashed lines in (b) indicates the full depletion voltage of the detector. Irradiation was carried out with proton of 45 $\mathrm{MeV}, 1 \times 10^{12} \mathrm{~cm}^{-2}$.

Fig. 2 shows $C_{j}$ versus $V_{R}$ and $1 / C_{j}^{2}$ versus $V_{R}$ for the detectors fabricated with different doping concentration of the substrates. We estimated the resistivity of the wafers from the 
$C-V$ data of non-irradiated samples. Estimated resistivities were $1.6,3.8,8.0$, and $15 \mathrm{k} \Omega . \mathrm{cm}$ for $\mathrm{S} 1, \mathrm{~S} 2, \mathrm{~S} 3$, and $\mathrm{S} 4$, respectively. The cross point of the two dashed line in Fig. 2 (b) shows an example of a full depletion voltage of the detector S4. The full depletion voltages of the detectors were $240,110,55$, and $27 \mathrm{~V}$ for S1, S2, S3, and S4, respectively when the thickness of the wafer was assumed to be $380 \mu \mathrm{m}$. From this information we can decide the resistivity of the wafer and operation voltage when the detector should be operated at full depletion mode.

While detectors irradiated with proton of $45 \mathrm{MeV}, 1 \times 10^{12}$ $\mathrm{cm}^{-2}$ show decreased junction capacitance and full depletion voltage. It is known to be due to the type conversion of some portion of substrate from n-type to p-type by irradiation [2].

\section{B. Mechanisms of Junction Leakage Current}

Performance of the semiconductor detector can be represented using leakage current as an indicator of a noise source in the detector. The reverse leakage current $\left(J_{R}\right)$ of the silicon p-n and PIN diodes is commonly described in literature as a combination of diffusion current and generation current components [3], [4]:

$$
J_{R}=J_{D i f f}+J_{G e n}=q \sqrt{\frac{D_{p}}{\tau_{p}}} \frac{n_{i}^{2}}{N_{D}}+\frac{q n_{i} W}{\tau_{e}}
$$

where $D_{p}$ is the diffusion coefficient, $\tau_{p}$ is the minority carrier lifetime, $W$ is the depletion width, $n_{i}$ is the intrinsic carrier concentration, $N_{D}$ is the doping concentration of the substrate, and $\tau_{e}$ is effective lifetime $\left(1 / \tau_{e}\right.$ is the generation rate in the depleted region).

The diffusion component is caused by the minority carriers generated in the neutral area, diffusing to the edge of the space charge region, and therefore it is independent of the applied reverse bias, at least until the full depletion is reached. Activation energy, $E_{a}$, of the diffusion current for thermal excitation is the band gap energy of the silicon, i.e. $1.1 \mathrm{eV}$.

If the generation centers are distributed uniformly, the generation current component is directly proportional to the depletion width, $W$, from which the generated carriers (minority and majority) are collected. The depletion width is given by

$$
W=\sqrt{\frac{2 \varepsilon_{s}}{q N_{D}}\left(V_{b i}+V_{R}\right)}
$$

Therefore $J_{G e n}$ is proportional to $\sqrt{V_{R}}$ and the activation energy of the generation current is generally 0.6 to $0.9 \mathrm{eV}$ depending on the energy level of the generation centers.

However another leakage current mechanism, called thermionic field emission (TFE) current, has been reported when the local electric field is higher than $0.1 \mathrm{MV} / \mathrm{cm}$ [5], [6]. Thermal emission rate from a trap level is enhanced by tunneling effect due to the strong electric field at the certain position of the depletion region. The activation energy of TFE current is generally lower than $0.5 \mathrm{eV}$. The particular feature of the current-voltage $(I-V)$ characteristics is that the leakage current increases exponentially as the applied voltage increases. Total leakage current of a PIN detector is the sum of $J_{\text {Diff, }} J_{\text {Gen }}$ and $J_{\text {TFE }}$.

\section{Measurement and Analysis of the Leakage Current}

Fig. 3 (a) shows measurement results of the leakage current for direct type detectors before irradiation. The changes of the leakage current after irradiation with proton of $45 \mathrm{MeV}, 1 \times 10^{12} \mathrm{~cm}^{-2}$ are shown in Fig. 3 (b).

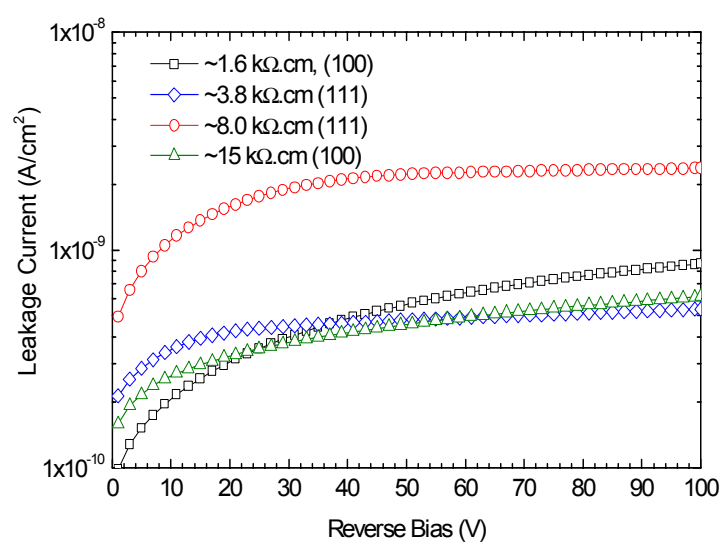

(a)

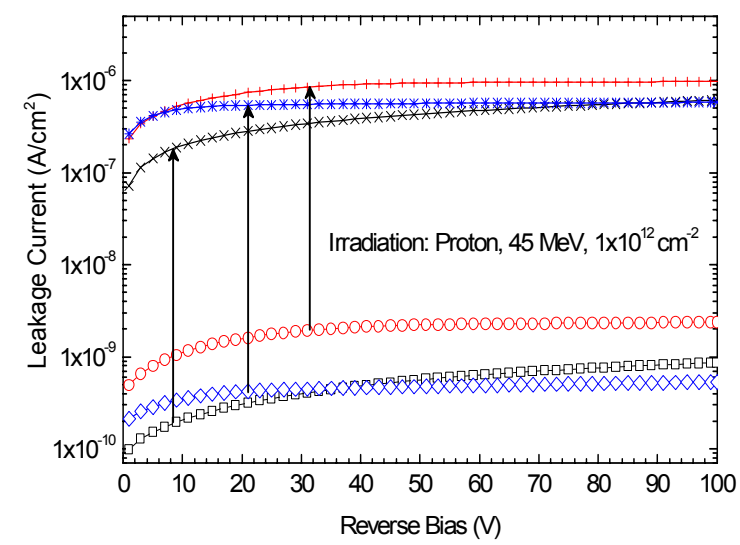

(b)

Fig. 3. Leakage current versus reverse bias curves: (a) before irradiation and (b) after irradiation with proton of $45 \mathrm{MeV}, 1 \times 10^{12} \mathrm{~cm}^{-2}$. Arrows in (b) indicate the change of leakage current after irradiation for each detector.

Fig. 3 gives us several features about the effects of the resistivity and crystal orientation on the leakage current. The important things are the following:

1. Substrate of (111) crystal orientation shows larger leakage current than (100) orientation. It is known to be due to the high interface trap density at the $\mathrm{Si} / \mathrm{SiO}_{2}$ interface.

2. High resistive substrate shows larger leakage current than low resistive substrate. From (3) and (4), we can see that the high resistive substrate flow more diffusion current due to the low $N_{D}$ and more generation current due to the wide depletion width.

3. At high reverse bias $(>35 \mathrm{~V})$, low resistive substrate shows larger leakage current than high resistive substrate for (100) orientation. It can not explained by the mechanism of diffusion current and generation 
current. We can explain it by the TFE current. The average electric field at the junction of the detector with $1.6 \mathrm{k} \Omega . \mathrm{cm}$ resistivity is about $3 \mathrm{kV} / \mathrm{cm}$, which is much lower than the criteria for the field emission current. However, if some defects exist in the depletion region especially at the $\mathrm{Si}_{-} \mathrm{SiO}_{2}$ interface, the local electric field can be increased above $0.1 \mathrm{MV} / \mathrm{cm}$ and the contribution of TFE current can be increased. Usually several folds of guard-rings should be designed around $\mathrm{p}+$ active to relieve the electric field at the Si$\mathrm{SiO}_{2}$ interface. It also can improve the breakdown characteristics of the detector. However guard-rings widen the depletion width at the surface and increase the generation current. Thus TFE current and generation current are at the trade-off relation from the viewpoint of depletion region width and electric field.

4. After proton irradiation, leakage current is enormously increased because of the increase of crystal defects. We can see that the leakage current of (100) orientation and (111) orientation are similar for the high resistive substrate after irradiation. It seems that the bulk defects by the irradiation dominate the leakage current rather than the trap at the $\mathrm{Si} / \mathrm{SiO}_{2}$ interface.

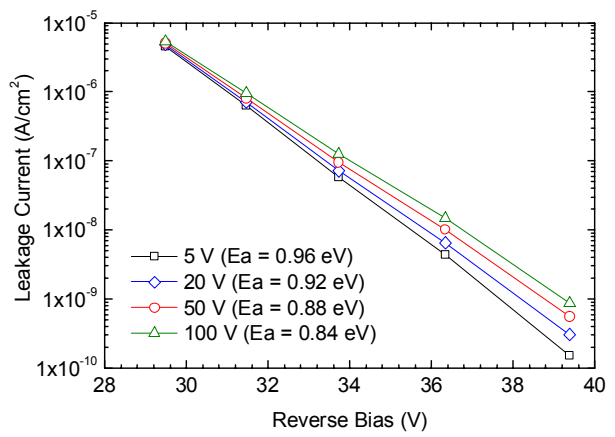

(a)

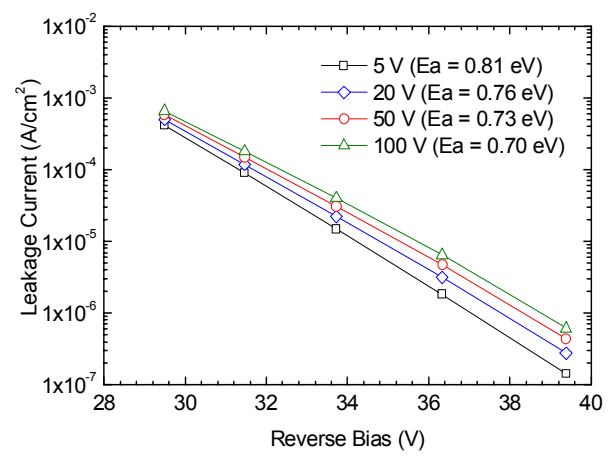

(b)

Fig. 4. An example of Arrhenius plot of the leakage current versus $1 / k T$ for the detector S1: (a) before irradiation and (b) after irradiation with proton of $45 \mathrm{MeV}, 1 \times 10^{12} \mathrm{~cm}^{-2}$. The slope of each curve is $-E_{a}$ for each condition.

Activation energy of the leakage current can be estimated from the temperature dependence of the leakage current. An Arrhenius plot of the leakage current versus $1 / k T$ yields the activation energy. We measured the leakage current at several temperatures, i.e. $25,50,75,100$, and $125^{\circ} \mathrm{C}$, and estimated the activation energy for several reverse bias from the Arrhenius plots.

TABLE II

ACTIVATION ENERGIES ESTIMATED FROM ARRHENIUS PLOT OF THE LEAKAGE CURRENT VERSUS $1 / k T$. ITALICS INDICATE IRRADIATED SAMPLES WITH PROTON OF $45 \mathrm{MeV}, 1 \times 10^{12} \mathrm{~cm}^{-2}$. UNIT OF THE ACTIVATION ENERGY IS $\mathrm{eV}$.

\begin{tabular}{l|c|c|c|c}
\multicolumn{1}{c|}{ Sample } & @5V & @20V & @50V & @100V \\
\hline S1, Non-irrad. & 0.96 & 0.92 & 0.88 & 0.84 \\
S1, Irrad. & 0.81 & 0.76 & 0.73 & 0.70 \\
\hline S3, Non-irrad. & 0.96 & 0.89 & 0.85 & 0.85 \\
S3, Irrad. & 0.74 & 0.70 & 0.67 & 0.67 \\
\hline S4, Non-irrad. & 0.94 & 0.91 & 0.90 & 0.89 \\
S4, Irrad. & 0.72 & 0.69 & 0.68 & 0.68
\end{tabular}

Fig. 4 shows an example of Arrhenius plot for the detector S1 and Table II summarized the estimated activation energy for all samples. At low reverse bias, the activation energies of the non-irradiated samples are about $0.95 \mathrm{eV}$ which is close to the activation energy of diffusion current. However, as the reverse bias increases, the activation energy is decreased. This means the portion of the generation current or TFE current increases as the depletion region width is increased at high reverse bias. On the other hand, the irradiated samples show reduced activation energies for overall reverse bias, which means irradiation make many generation centers, i.e. defects.

\section{Radiation Response Characteristics}

The amount of charge generation and collection for the radiation is another important factor of the radiation detector together with leakage current. To compare the radiation response characteristics, we exposed the X-ray beam of $60 \mathrm{kV}$ tube voltage to each detector and read the output current. The reverse bias of $5 \mathrm{~V}$ or $100 \mathrm{~V}$ was forced onto the detectors.

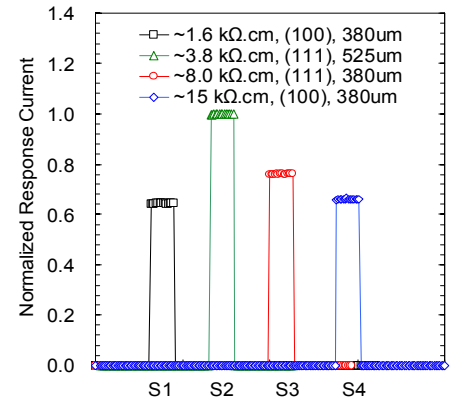

(a)

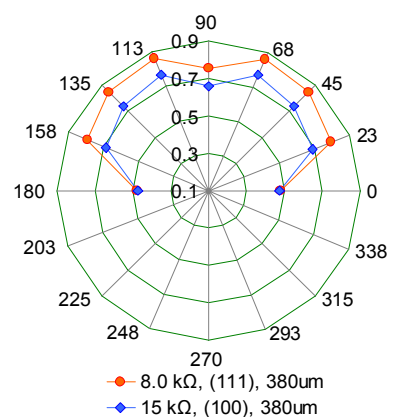

(b)
Fig. 5. (a) Normalized output current of the detectors and (b) directional response for irradiation by $\mathrm{X}$-ray of $60 \mathrm{kV}$ tube voltage.

Fig. 5 shows the normalized output current for the X-ray exposure and directional response of the area monitor. It is known that the charge collection efficiency (i.e. ratio of mean number of electrons measured vs. mean number of electrons generated) of the (100) oriented wafers are preferred over (111) wafers [7]. However, in our experiments the detector with (111) oriented substrate shows about $20 \%$ higher output 
current than (100) orientation. We think it is due to the higher carrier generation for (111) oriented wafers in spite of lower charge collection efficiency, because the atomic density along the (111) plane is higher than (100) plane. The output current is independent on the resistivity of the wafers and also on the operation voltage. This means the output current by X-ray exposure is independent on the depletion width and the carriers generated beyond the depletion region also contribute to the output current by the diffusion of the carriers. Carrier lifetime of the all wafer is the order of a few msec which is sufficient to diffuse into the depletion region from the neutral region. It is also shown that the most influential factor on the output current is the thickness of the wafer. Fig. 4 (b) shows the directional response of the detector for the X-ray exposure. The directional response has a deviation of $\pm 12 \%$ in the directional range of 70 degrees.

Finally, we assembled one of our detectors with amplifier circuit for the application of $\gamma$ ray dosimeter and tested whether it can detect the $\mathrm{Cs}_{137}$ natural $\gamma$ ray. Selected sample was S3 of which leakage current is relatively low at low reverse bias and the radiation response characteristics is better than the other samples. Fig. 6 (a) shows the architecture of the pulse mode signal processing circuit for the $\gamma$ ray dosimeter. Fig. 6 (b) is a photograph of our dosimeter and Fig. 6 (c) shows our detector is very sensitive to the $\mathrm{Cs}_{137}$ natural $\gamma$ ray.

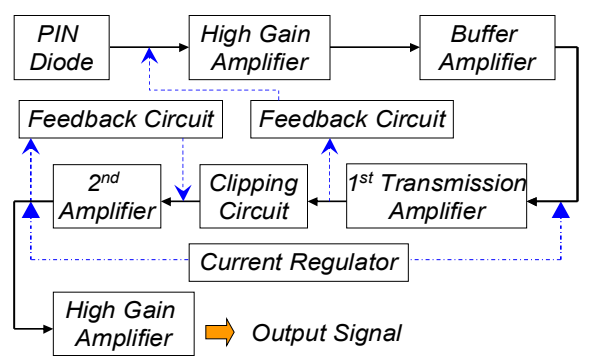

(a)

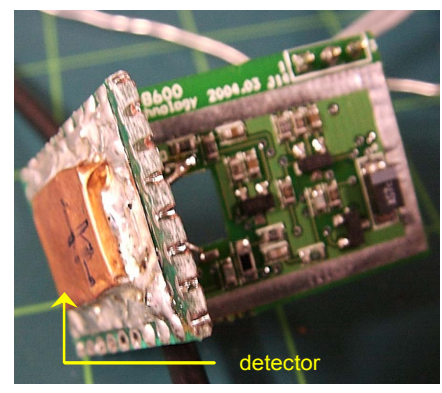

(b)

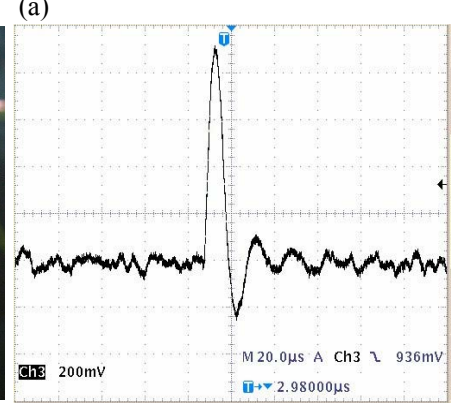

(c)
Fig. 6. (a) Architecture of the pulse mode signal processing circuit for the $\gamma$ ray dosimeter, (b) a photograph of the dosimeter, and (c) test result for the $\mathrm{Cs}_{137}$ natural $\gamma$ ray. The detector in (b) is wrapped with metal foil.

\section{CONCLUSIONS}

We have fabricated radiation detectors with high resistive ntype silicon wafers having different resistivity and crystal orientation, and studied on the leakage current and radiation response characteristics. The detector with (111) oriented substrate shows higher leakage current than (100) orientation and high resistive substrate shows larger leakage current than low resistive one. In case of (100) oriented substrate, the leakage current of low resistive substrate is smaller than high resistive one below the reverse bias of $35 \mathrm{~V}$, but it is contrary above $35 \mathrm{~V}$. From the consideration on the leakage current mechanisms, we can see that most leakage current is diffusion current at low reverse bias voltage. However, as the reverse bias voltage increases, the portion of the generation current and TFE current is increased. After proton irradiation, the increased leakage current is generation current at both low and high reverse bias.

To compare the charge generation and collection for the radiation, we exposed the detectors to the X-ray beam and read the output current. The detector with (111) oriented substrate shows $20 \%$ higher output current than (100) orientation and it is independent on the resistivity of the wafers. The most influential factor on the output current is the thickness of the wafer. From the results we can suggest a high resistive, (100) oriented and thick wafer for the direct type radiation detector, and a low resistive and thin wafer for indirect type radiation detector. Finally we assembled our detector with read-out integrated circuit for the application of $\gamma$ ray dosimeter and tested whether it can detect the $\mathrm{Cs}_{137}$ natural $\gamma$ ray. Our detector is very sensitive to it.

\section{ACKNOWLEDGMENT}

We thank president Hyun-Sik Chae of SF Technology Co. Ltd. for assembling our detectors with amplifier circuit.

\section{REFERENCES}

[1] P. Rehak, "Silicon radiation detectors," IEEE Trans. Nucl. Sci., vol. 51, no. 5, pp. 2492-2497, Oct. 2004.

[2] G. Calefato, D. Creanza, M. de Palma, L. Fiore, S. My, V. Radicci, G. Selvaggi, P. Tempesta, M. M. Angarano, G. M. Bilei, M. Biasini, M. Giorgi, O. Militaru, and L. Servili, "A comparison on radiation tolerance of $<100>$ and $<111>$ silicon substrates of microstrip detectors," Nucl. Instrum. Methods, vol. A476, pp. 744-750, 2002.

[3] S. M. Sze, Physics of Semiconductor Devices, 2nd ed. New York: Wiley, 1981.

[4] A. Ruzin and S. Marunko, "Current mechanisms in silicon PIN structures processed with various technologies," Nucl. Instrum. Methods, vol. A492, pp. 411-422, 2002.

[5] K. Ohyu, M. Ohkura, A. Hiraiwa, and K. Watanabe, "A mechanism and a reduction technique for large reverse leakage current in p-n junctions," IEEE Trans. Elec. Dev., vol. 42, pp.1404-1412, Aug. 1995.

[6] T. Hamamoto, S. Sugiura, and S. Sawada, "On the retention time distribution of dynamic random access memory (DRAM)," IEEE Trans. Elec. Dev., vol. 45, pp.1300-1309, Jun. 1998.

[7] M. Popp, R. Hartmann, H. Soltau, L. Struder, N. Meidinger, P. Holl, N. Krause and C. Von Znathier, "Modeling the energy response of pnCCDs in the 0.2-10 keV band," Nucl. Instrum. Methods, vol. A439, pp. $567-574,2000$. 